\title{
Wie ist ein nachhaltiger Umgang mit Plastik möglich?
}

\section{Eine Vorstellung der inter- und transdisziplinär arbeitenden Nachwuchsgruppe „PlastX“}

\section{Johanna Kramm und Carolin Völker}

\section{Zusammenfassung}

Stellt Plastik ein Risiko für unsere Umwelt dar? Wie finden wir einen besseren Umgang mit einem Material, das so viele Bereiche unseres Alltags durchdrungen hat? Mit diesen und weiteren Fragen beschäftigt sich die interund transdisziplinär arbeitenden Nachwuchsgruppe „PlastX - Plastik in der Umwelt als systemisches Risiko“. Der Beitrag stellt die unterschiedlichen Forschungsfelder (Mikroplastik, Meeresmüll, Verpackungen und Bioplastik) vor. Der Ansatz der systemischen Risiken wird als integrative Perspektive diskutiert.

\section{$1 \quad$ Einleitung}

Stellt Plastik ein Risiko für unsere Umwelt dar? Wie finden wir einen besseren Umgang mit einem Material, das so viele Bereiche unseres Alltags durchdrungen hat? Mit diesen und weiteren Fragen beschäftigen wir uns in unserer inter- und transdisziplinär arbeitenden Nachwuchsgruppe „PlastX - Plastik in der Umwelt als systemisches Risiko“, die am ISOE - Institut für sozial-ökologische Forschung

\footnotetext{
J. Kramm $(\varangle) \cdot$ C. Völker

ISOE - Institut für sozial-ökologische Forschung in Frankfurt am Main, Frankfurt am Main, Deutschland

E-Mail: kramm@isoe.de

C. Völker

E-Mail: voelker@isoe.de
} 
beheimatet ist. Die Soziale Ökologie als disziplinübergreifende Nachhaltigkeitsforschung versucht durch naturwissenschaftliche Methoden und Konzepte zu verstehen, in welchem Ausmaß Boden, Wasser und Luft verschmutzt sind und inwieweit diese Verschmutzungen Ökosysteme gefährden. Gleichzeitig werden mithilfe sozialwissenschaftlicher Methoden und Konzepte die gesellschaftlichen Ursachen und Verursacher von Umweltproblemen betrachtet; damit rücken die komplexen Wechselbeziehungen zwischen Gesellschaft und Natur in den Fokus der Forschung. Zudem ist diese Art der Forschung transdisziplinär, das heißt, sie orientiert sich an gesellschaftlichen Problemen und ist praxisbezogen (Becker und Jahn 2006).

Wir, eine Natur- und eine Sozialwissenschaftlerin, leiten gemeinsam das Team aus insgesamt sechs Nachwuchswissenschaftler*innen, die die Disziplinen Humangeografie, Ökotoxikologie, Chemie und Soziologie vereinen. Forschungspartner sind die Goethe-Universität Frankfurt und das Max-Planck-Institut für Polymerforschung in Mainz. Gefördert wird unsere Nachwuchsgruppe vom Bundesministerium für Bildung und Forschung (BMBF). Im Förderprogramm „Forschung für nachhaltige Entwicklungen“ (FONA) wurde gezielt der Förderbereich „Nachwuchsgruppen in der Sozialökologischen Forschung“ aufgelegt, um jungen Wissenschaftler*innen die Möglichkeit zu geben, sich im Bereich Nachhaltigkeitsforschung zu qualifizieren und die inter- und transdisziplinäre Forschung in der deutschen Hochschullandschaft zu stärken. Eine zentrale Herausforderung hierbei ist, dass wissenschaftliche Qualifizierung an den Universitäten disziplinär ausgerichtet ist, $d$. h. dass trotz inter- und transdisziplinärer Arbeit eine disziplinäre Profilierung erreicht werden muss. Des Weiteren stehen bei transdisziplinär arbeitenden Nachwuchsgruppen nicht nur wissenschaftliche Publikationen als Ergebnis im Mittelpunkt, sondern auch Produkte für die gesellschaftliche Praxis, z. B. in Form von Handlungsleitfäden oder Politikempfehlungen. Mit dem interund transdisziplinären Zugang stellt sich unsere Forschungsgruppe also gleich zwei zentralen Integrationsherausforderungen: Zum einen eine gute Zusammenarbeit von Wissenschaftler*innen unterschiedlicher Disziplinen zu gewährleisten sowie Spannungen zwischen natur- und sozialwissenschaftlichen Perspektiven zu überwinden und zum anderen eine gute Einbindung von praktischem Wissen in unserer Forschung zu erreichen (für eine Diskussion der zentralen Herausforderungen siehe Jaeger-Erben et al. 2018). Unterstützt wird dies durch unseren Projektbeirat, in dem sowohl erfahrene Wissenschaftler*innen als Mentor*innen für die Gruppenmitglieder dienen als auch Partner aus der Praxis ihre Erfahrungen und Erkenntnisse in die Forschung eintragen.

Unser Forschungsthema Plastik in der Umwelt stellt kein neuartiges Problem dar, sondern wird bereits seit den 1970er Jahren gesellschaftlich diskutiert 
(Kramm und Völker 2018). Dennoch ist das Problem bis heute nicht gelöst und die Debatte aktueller als je zuvor: Immer mehr wissenschaftliche Studien verweisen auf die wachsende Menge an Plastikmüll in Meeren und Ozeanen, der, zersetzt zu kleinsten Partikeln (,Mikroplastik“), nahezu alle Ökosysteme kontaminiert. Gleichzeitig ist Plastik heute noch weniger aus dem alltäglichen Leben wegzudenken als damals; die weltweite Produktion ist allein zwischen 2005 und 2015 von 230 auf 320 Mio. Tonnen angestiegen (PlasticsEurope 2016). Plastik ist günstig herstellbar, gut formbar, leicht und beständig und bietet deshalb viele Vorteile gegenüber anderen Materialien. Letztendlich sind es jedoch genau diese Vorteile, die zu den unerwünschten Effekten in der Umwelt führen - die massenhafte Verwendung führt zu einem hohen Abfallaufkommen und zum Eintrag in die Umwelt und die Beständigkeit des Materials setzt sich auch in der Umwelt fort, was bedeutet, dass die meisten Kunststoffe dort akkumulieren und kaum oder nur sehr langsam abgebaut werden. Diese Ambivalenz verdeutlicht, dass es sich bei dem Thema nicht um ein rein wissenschaftliches, sondern um ein komplexes lebensweltliches Problem handelt. Neben den Umweltfolgen, die die massenhafte Plastikverwendung mit sich bringt, muss auch die alltagspraktische Bedeutung des Materials betrachtet werden, da nur so die Ursachen des Problems langfristig behoben werden können. Bislang sind die Auswirkungen von Plastik auf die Umwelt und die menschliche Gesundheit wissenschaftlich nicht ausreichend beschrieben und es herrschen widerstreitende Ansichten darüber, ob und wie der Plastikeintrag in die Umwelt verringert werden kann. In unserer Forschungsgruppe nehmen wir eine breite, möglichst ganzheitliche Sicht ein und rahmen die Problematik als „systemisches Risiko“. Damit möchten wir das Phänomen „Plastik in der Umwelt“ über die möglichen Umweltwirkungen hinaus in seiner Komplexität verstehen, verschiedene Lösungsansätze tiefer gehend untersuchen und ihre Wechselwirkungen verstehen. Die konzeptionelle Rahmung der Problematik wird im nächsten Abschnitt näher vorgestellt.

\section{$2 \quad$ Plastik in der Umwelt als systemisches Risiko}

Der Begriff „,systemisches Risiko“ stammt ursprünglich aus der Finanzwissenschaft und bezeichnet dort das Risiko, dass durch die Zahlungsunfähigkeit eines Marktteilnehmers andere Markteilnehmer ebenfalls in Mitleidenschaft gezogen werden, was schließlich den funktionellen Zusammenbruch des Finanzsystems bewirken kann (Bechmann et al. 2007). In den letzten Jahren wurde der Begriff auch außerhalb der Finanzwissenschaft verwendet und vor allem in 
der sozial-ökologischen Forschung weiterentwickelt. Ausgang war das OECDProjekt „Emerging Risks in the 21st Century: An Agenda for Action“ (OECD 2003), in dem systemische Risiken als katastrophale Ereignisse wie Naturkatastrophen oder Infektionskrankheiten beschrieben werden, die das Potenzial besitzen, ganze Systeme und Infrastrukturen, von denen Gesellschaften abhängig sind, zu gefährden. Zwar stellt der Eintrag von Plastik in die Umwelt kein singuläres, katastrophales Ereignis dar, doch nimmt die zunehmende Verschmutzung Einfluss auf zahlreiche Ökosysteme und verschiedene gesellschaftliche Bereiche. Der Plastikeintrag geschieht dabei dezentral und fortwährend durch die täglich anfallenden Plastikabfälle, die teilweise (un)beabsichtigt in die Umwelt gelangen. Dieser Plastikabfall - ca. $63 \%$ davon sind Verpackungen (Europäische Kommission 2013) - stammt aus unterschiedlichen Bereichen des alltäglichen Lebens, der Konsum der Plastikprodukte erfolgt zum Großteil allerdings eher unbewusst: Lebensmittelverpackungen bedienen keine Nachfrage nach Plastik, sondern nach frischen Lebensmitteln, in der Medizin garantieren Plastikverpackungen sterile Produkte und mit Plastiktüten werden Einkäufe transportiert (Andrady et al. 2015; Andrady und Neal 2009). In der sozial-ökologischen Forschung ändert sich also die Analyseperspektive: Im Fokus stehen die normalen, installierten und routinierten Abläufe in einer Gesellschaft, z. B. der Lebensmittelversorgung, die zu einer kontinuierlichen, kumulativen Schadensproduktion führen und somit die verbundenen Ökosysteme sowie die Gesellschaft selbst gefährden können. Anders ausgedrückt wird also untersucht, wie gerade der „Normalbetrieb“ moderner Gesellschaften zu unerwünschten Nebenfolgen und damit zu kontinuierlicher Risikoproduktion führt (Bechmann et al. 2007).

Doch wie hoch ist nun das Risiko, welches von Plastik für die Umwelt ausgeht? Viele wissenschaftliche Studien zeigen, dass sich wildlebende Meerestierarten in größerem Plastikabfall verheddern oder Plastikfragmente verschlucken können (Wright et al. 2013). Doch ob die verschluckten Plastikteile toxisch auf die Organismen wirken und welche Folgen der eingebrachte Plastikabfall letztendlich für das gesamte Ökosystem hat, kann wissenschaftlich bisher nicht ausreichend bewertet werden. Da Plastik keine einheitliche chemische Substanz ist, sondern ein Werkstoff, der aus verschiedenen Polymeren mit unterschiedlichen Zusatzstoffen wie Weichmachern, Flammschutzmitteln oder Farbstoffen besteht, ist die Untersuchung der biologischen Effekte äußerst komplex und Gegenstand hoher Unsicherheit. Es existiert zudem kein vollständiger Überblick über Qualität und Quantität der in der Umwelt vorkommenden Plastikarten, die zusätzlich durch Umwelteinflüsse in ihrer chemischen Zusammensetzung verändert werden. Der klassische Ansatz, der zur Umweltrisikobewertung von 
Chemikalien entwickelt wurde, eignet sich aufgrund dieser komplexen Zusammensetzung nicht, um ,sichere“ oder ,gefährliche“ Konzentrationen von Plastik in der Umwelt zu bestimmen (Kramm und Völker 2018). Zudem sind die ökologischen Folgen nicht die einzigen Risiken, die im Zusammenhang mit Plastik in der Umwelt betrachtet werden müssen. In vielen Fällen aus ästhetischen Gründen, führen die Plastikabfälle auch zu politischen, sozialen und wirtschaftlichen Folgen, z. B. wenn die Gefahr besteht, dass durch verschmutzte Strände Tourismuseinnahmen verloren gehen (Ballance et al. 2000; Jang et al. 2014) oder Fischer Einkommensverluste durch Plastikmüll erleiden (Nash 1992). Ausgelöst durch mediale Berichterstattungen über umstrittene Studien (Der Spiegel 2013; NDR 2014), wird Mikroplastik in der öffentlichen Debatte auch als Risiko für die Lebensmittelversorgung wahrgenommen (Bundesinstitut für Risikobewertung (BfR) 2016). Plastik in der Umwelt entfaltet also Wirkungen jenseits der Ökosysteme, in die es gelangt. Die dezentrale Risikoproduktion und die vielfältigen Auswirkungen unterstreichen, dass sich die Problematik nicht als klassisches Risiko mit Hilfe von Eintrittswahrscheinlichkeiten und Schadensausmaß kalkulieren lässt (Kramm et al. 2018). Folglich existieren auch keine einfachen, optimalen Lösungsstrategien, sondern der gesellschaftliche Umgang mit Plastik muss in vielen Bereichen grundsätzlich anders gestaltet werden. In der Debatte treffen allerdings völlig verschiedene Akteure aufeinander, die Risikoproduzenten oder Betroffene - oder beides gleichzeitig - sein können (für die Akteure Wissenschaft und Medien siehe Völker et al. 2019). Einerseits aufgrund unterschiedlicher Wissensbestände, Wertvorstellungen und Interessen, andererseits aber auch aufgrund der nicht eindeutigen wissenschaftlichen Datenlage, nehmen die Akteure das Problem unterschiedlich wahr, was zu widerstreitenden Ansichten hinsichtlich möglicher Lösungsstrategien führt. So unterstreichen einige die Risiken des Materials und unterstützen dessen Vermeidung sowie die Verwendung alternativer Materialien. Andere halten nicht Plastik als Material für problematisch, sondern lediglich dessen falsche Entsorgung. Als mögliche Lösungsstrategien liegen dabei ganz verschiedene Ansätze auf dem Tisch, die über besseres Recycling, effizienteres Abfallmanagement, Bewusstseinsbildung, nachhaltigeren Konsum, abbaubare Plastikarten, bis zum Verbot bestimmter Plastikprodukte und zu Müllsammelaktionen reichen. Wer letztendlich welche Rolle bei der Umsetzung der jeweiligen Lösungsansätze übernehmen muss - sei es die Politik, Akteure aus der Wirtschaft, gemeinnützige Organisationen oder der ,,verantwortliche Konsument“ - ist eine weitere Frage, über die gegensätzliche Meinungen herrschen.

Zusammengefasst lässt sich Plastik in der Umwelt also durch folgende Charakteristika als systemisches Risiko beschreiben: Die Auswirkungen sind nicht lokal 
begrenzt, sondern können von einem System (z. B. dem Ökosystem) in ein anderes System (z. B. den Tourismus) ausstrahlen. Weitere Kennzeichen sind eine hohe Komplexität der Ursache-Wirkungs-Ketten sowie ein hohes Maß an Unsicherheit und Ambiguität (Mehrdeutigkeit) hinsichtlich der zu erwartenden Konsequenzen (Kramm und Völker 2018). Deutlich wird, dass nicht nur ein Lösungsweg verfolgt werden kann, sondern im Sinne einer ganzheitlichen Betrachtung in verschiedenen Handlungsfeldern angesetzt werden muss. Für unsere Forschung haben wir deshalb unterschiedliche Handlungsfelder identifiziert und Fragestellungen abgeleitet, die zu einem besseren Verständnis der Problematik führen sowie verschiedene Lösungsansätze näher betrachten sollen. Erstens untersuchen wir die Effekte, die in die Umwelt eingebrachtes Plastik auf die dort lebenden Organismen hat und tragen dazu bei, das Risiko für Ökosysteme einschätzen zu können. Wir konzentrieren uns dabei auf die Auswirkungen von Mikroplastik und führen ökotoxikologische Untersuchungen mit Wasserorganismen durch. Zudem möchten wir den klassischen Ansatz zur Umweltrisikobewertung von Chemikalien weiterentwickeln, um daraus adäquate Managementoptionen für Mikroplastik in der Umwelt abzuleiten. Die zweite Frage beschäftigt sich mit dem Thema Meeresmüll als globalisiertes Umweltproblem und seiner politischen Bearbeitung. Hier untersuchen wir, wie der weitere Eintrag von Plastikmüll in die Weltmeere verringert werden kann, und analysieren unterschiedliche Managementstrategien, die dieses Problem aufgreifen. Im dritten Forschungsfeld geht es um die Strategie, die in der Abfallhierarchie an oberster Stelle steht, der Vermeidung oder Reduktion der Kunststoffverwendung. Ein Großteil der produzierten Kunststoffe wird für Verpackungen verwendet, diese gelangen zudem am häufigsten in die Umwelt. Wir untersuchen Konsum- und Produktionsmuster von Plastikverpackungen im Lebensmittelbereich und gehen der Frage nach, wie deren Verwendung nachhaltiger zu gestalten ist. Die vierte Frage, die direkt an das dritte Forschungsfeld anschließt, widmet sich möglichen Verpackungsalternativen und der Substitution konventioneller Kunststoffarten. Hierfür werden neue Polymere im Labor synthetisiert, die als Ersatzstoff für herkömmliche Kunststoffe in Verpackungen dienen können und gleichzeitig in der Umwelt besser abbaubar sein sollen.

Das Wissen aus den verschiedenen Forschungsfeldern ist essenziell, da nur so eine Gesamtbetrachtung der Problematik möglich ist und Lösungsstrategien mitsamt ihrer (unerwünschten) Nebenfolgen abgewogen werden können. Aus der systemischen Perspektive werden die in den unterschiedlichen Forschungsfeldern entwickelten Handlungsoptionen integriert betrachtet. Hier wird erarbeitet, wie komplexe Umweltprobleme mit naturwissenschaftlichen Methoden untersucht werden können und wie diese Probleme gesellschaftlich und politisch 
bewertet werden. Im Folgenden werden die unterschiedlichen Forschungsbereiche detaillierter vorgestellt.

\section{1 Ökotoxikologische Bewertung von Mikroplastik:}

Wie ist das Umweltrisiko von Mikroplastik einzuschätzen?

Stellt Mikroplastik ein Risiko für das Ökosystem dar? Diese Frage wird inzwischen vielfach diskutiert und es gibt bisher noch keine abschließende Antwort. Eine wachsende Zahl von Studien belegt das Vorkommen der kleinen Plastikfragmente mit einer Größe von unter 5 Millimetern in nahezu allen aquatischen Ökosystemen. Mikroplastik entsteht, wenn größerer Plastikabfall durch Umwelteinflüsse wie Sonnenlicht oder mechanische Prozesse in kleinere Fragmente zersetzt wird (Andrady 2011). In diesem Fall spricht man von sekundärem Mikroplastik. Primäres Mikroplastik bezeichnet Plastikpartikel, die direkt als Partikel angewendet werden, z. B. Pellets zur Plastikproduktion oder Partikel in Körperpflegeprodukten, und durch ihren Gebrauch ebenfalls in die Umwelt gelangen können (Zitko und Hanlon 1991; Gregory 1996). Zunächst vor allem in der marinen Umwelt detektiert, wird es nun auch vermehrt in limnischen Gewässern, also Seen und Flüssen, nachgewiesen (Wagner et al. 2014).

Doch wie wirken sich die Plastikpartikel auf die Lebewesen in den Gewässern aus? Bisher zeigt sich, dass Mikroplastik in der Umwelt von verschiedenen Wasserorganismen aufgenommen wird, darunter einige Fischarten, Krebstiere, Schnecken und Würmer (Eerkes-Medrano et al. 2015). In vielen Fällen werden die Partikel nach der Aufnahme wieder ausgeschieden, ohne dass sie toxisch auf die Organismen wirken. Aus einigen Laborexperimenten geht jedoch auch hervor, dass sich nach der Aufnahme von Mikroplastikpartikeln toxische Wirkungen auf verschiedene Organe und eine erhöhte Sterblichkeit der Organismen zeigen (Oliveira et al. 2013; Rehse et al. 2016; Lu et al. 2016). Unklar ist, über welche Wirkmechanismen diese Effekte in den Organismen zustande kommen. Es wird vermutet, dass Mikroplastik mit Nahrung verwechselt wird, bei einer Aufnahme den Magen-Darm-Trakt verstopft und so zu einer verringerten Nahrungsaufnahme der Organismen führt, die dann letztendlich die beobachteten schädlichen Effekte verursacht (z. B. Besseling et al. 2014). Weiterhin könnten Chemikalien, die den Kunststoffen bei der Herstellung zugesetzt werden (z. B. Weichmacher), aus den Partikeln auslaugen und für einen toxischen Effekt im Organismus verantwortlich sein. Auch wird vermutet, dass andere Umweltchemikalien an Mikroplastik anheften und über die Partikel in die Organismen transportiert werden (Teuten et al. 
2007). Letztendlich ist allerdings unklar, ob diese Effekte tatsächlich umweltrelevant sind, da die Versuche zumeist mit sehr hohen Partikelkonzentrationen durchgeführt wurden, die so in der Umwelt nicht vorkommen (Koelmans et al. 2017).

Um die Umweltwirkung von Mikroplastik einschätzen zu können, fehlt es einerseits noch an wissenschaftlichen Studien, die sowohl einen Überblick darüber geben, welche Partikel in welchen Mengen in der Umwelt vorhanden sind (Browne et al. 2011), als auch an Studien zu den biologischen Effekten, insbesondere Langzeiteffekten bei chronischer Exposition (Wagner et al. 2014). Bereits durchgeführte Studien sind zudem oft nicht vergleichbar, da keine einheitlichen Methoden verwendet wurden, um die Partikel in der Umwelt und die Effekte zu charakterisieren. Andererseits existiert, wie bereits erwähnt, bisher kein tragfähiges Konzept, das Umweltrisiko dieser Stoffgruppe zu bewerten. Der herkömmliche Ansatz wird der Vielzahl der Mikroplastikpartikel, die sich in Form, Größe, chemischer Zusammensetzung und ihrer Herkunft unterscheiden, nicht gerecht, was die Prognose der Umweltauswirkungen erschwert.

In PlastX gehen wir der Frage nach, wie die Auswirkungen von Mikroplastik auf Gewässerökosysteme bewertet werden können. Wichtige Fragen sind: Was passiert mit den Plastikpartikeln nach der Aufnahme in den Organismus? Werden sie auf bestimmte Gewebe übertragen oder sofort ausgeschieden? Verändert Mikroplastik das Fressverhalten der Organismen oder löst es Entzündungsreaktionen aus? Wie beeinflussen Faktoren wie Polymertyp, Größe und Form die Toxizität? Haben die zahlreichen Zusatzstoffe (wie z. B. Weichmacher) Auswirkungen auf aquatische Organismen? Um diese Fragen zu beantworten, werden im Labor verschiedene Experimente mit Wasserorganismen durchgeführt, die gegenüber Mikroplastikpartikeln exponiert werden (Zimmermann et al. 2020).

Weiterhin wird erarbeitet, wie bestehende Konzepte zur Umweltrisikobewertung von chemischen Substanzen angepasst werden müssen, um das Gefahrenpotenzial von Mikroplastik adäquat einzuschätzen. Bei der Umweltverträglichkeitsprüfung werden mögliche Exposition und Effekte einer Substanz bewertet, um das Risiko zu charakterisieren und entsprechende regulatorische und politische Maßnahmen zur Risikominimierung zu ergreifen (European Chemicals Bureau 2003). Kann das Umweltrisiko bewertet werden, erleichtert dies Handlungsempfehlungen und, falls nötig, nationale bzw. europaweite Regulationen des Mikroplastik-Eintrages (Wagner et al. 2014). 


\subsection{Der Ozean als globale gemeinsame Ressource:}

Mit welchen Maßnahmen lässt sich Meeresmüll wirksam begegnen?

Unabhängig davon, ob kleinste Plastikpartikel ein ökotoxikologisches Risiko für die Umwelt darstellen, herrscht Konsens, dass der Eintrag von Plastikabfällen in die Umwelt reduziert werden muss. Durch medienwirksame Bilder, die auf verschmutzte Strände, treibenden Plastikmüll und beliebte Meerestierarten, die sich in Plastikmüll verheddern, hinweisen, ist insbesondere das Thema Meeresmüll in den letzten Jahren zunehmend in den Fokus der öffentlichen und politischen Aufmerksamkeit gerückt (Derraik 2002; UNEP 2016; StöfenO'Brien 2015). Es wird geschätzt, dass heute etwa dreiviertel des Meeresmülls aus Plastikabfällen besteht und dass es sich dabei um ca. 140 Mio. Tonnen handelt, die entweder im Meer treiben oder auf den Boden abgesunken sind (Jambeck et al. 2015; MacArthur et al. 2016; PlasticsEurope 2016). Diese Plastikabfälle gelangen durch unsachgemäße Entsorgung, unzureichend gemanagte Deponien, fehlendes Abfall- oder Abwassermanagement, aber auch touristische Aktivitäten über Flüsse, Niederschlagswasser und Wind in die Ozeane (UNEP 2016; Jambeck et al. 2015). Insbesondere asiatische Länder werden wegen ihrer hohen Bevölkerungsdichte, dem vielfach ungeordneten Abfallmanagement sowie den langen Küstenlinien für den Eintrag verantwortlich gemacht (Jambeck et al. 2015). In Schwellenländern wie Indien und China, aber auch in weiteren asiatischen Staaten mit einem hohen Wirtschaftswachstum wie Vietnam oder Thailand, bilden sich konsumstarke Bevölkerungsschichten heraus, was zu einer höheren Nachfrage von Plastikprodukten führt, seien es in Plastik verpackte Lebensmittel oder auch Haushaltswaren und hochwertige Konsumgüter. Wachsende Produktion und zunehmender Konsum stehen oft einem unzureichenden Abfall- und Abwasserregime gegenüber. Zugleich tragen Exporte von Plastikmüll, z. B. aus der EU nach Asien, zu einer Verschärfung des Müllproblems bei (Velis 2014). Plastikabfälle in Ozeanen werden mit einer Reihe negativer Auswirkungen assoziiert; neben den ökologischen Folgen werden, z. B. Navigationsschwierigkeiten für Schiffe durch treibenden Müll sowie Einkommenseinbußen in der Tourismusbranche aufgrund verschmutzter Strände angeführt (Derraik 2002; Gregory 2009; UNEP 2016). Der Meeresmüll ist ein grenzüberschreitendes Problem, so können Strände durch Müll verschmutzt werden, der durch die Meeresströmungen von weit her angespült wurde. Das heißt auch, und hier gibt es Ähnlichkeiten zum Klimawandel, dass Verursacher und Leidtragende oft nicht zusammenfallen. Das Meer ist eine globale gemeinsame Ressource (WBGU 2013) und sollte als „Erbe der Menschheit“, wie es im Seerechtsübereinkommen der Vereinten Nationen (UN-CLOS) angelegt ist, geschützt werden. 
In den vergangenen Jahren wurden internationale Strategien verabschiedet, die den Umgang mit Plastikabfall in Ozeanen regulieren sollen. So hat die globale Gemeinschaft mehrere Übereinkommen zum Schutz der Meere und Ozeane verabredet, in der auch Plastikabfälle eine Rolle spielen, darunter z. B. das Internationale Übereinkommen von 1973 zur Verhütung der Meeresverschmutzung durch Schiffe (MARPOL) und das Seerechtsübereinkommen der Vereinten Nationen (UNCLOS) sowie regionale Übereinkommen wie die MeeresstrategieRahmenrichtlinie (MSRL) der Europäischen Union. Aber auch Akteure aus Wissenschaft, Umweltverbänden und Entwicklungszusammenarbeit nehmen eine aktive Rolle ein, wenn es um den Meeresschutz geht (Kerber und Kramm 2020). So widmen sich globale Umweltorganisationen sowie Organisationen der Entwicklungszusammenarbeit zunehmend dem Thema Meeresmüll mit einem internationalen Blick. Die weiterhin zunehmende Verschmutzung der Ozeane zeigt jedoch, dass diese multilateralen Vereinbarungen Grenzen haben, da diese auch in nationale und lokale Maßnahmen umgesetzt werden müssen.

Uns beschäftigt daher, wie Plastikabfälle in Ozeanen als grenzüberschreitendes Umweltproblem politisch bearbeitet werden und wie bestimmte multilaterale Übereinkommen und nationale Politikstrategien in konkreten Maßnahmen umgesetzt werden. In Zusammenarbeit mit zwei Organisationen der internationalen Zusammenarbeit aus Umwelt und Entwicklung untersuchen wir in Fallstudien in Vietnam, welche lokalen Herausforderungen im Abfallmanagement bestehen, wie der Meeresmüll wahrgenommen wird und welche Ansatzpunkte für Maßnahmen bestehen (Kerber und Kramm 2021). Forschungsfragen umfassen: Wie gestaltet sich die Problemwahrnehmung von unterschiedlichen Akteuren vor Ort? Welche Rolle spielen Kunststoffe und Plastikmüll in Alltagspraktiken? Wie werden nationale Politikstrategien in konkreten Maßnahmen umgesetzt? Die Forschungsergebnisse werden den Partnerorganisationen zurückgespielt, um Projekte zur Vermeidung von Meeresmüll zu verbessern.

\subsection{Kunststoffe in der Lebensmittelversorgung:}

Wie kann das Verpackungsaufkommen reduziert werden?

Um den Eintrag von Plastikabfällen in die Umwelt grundsätzlich zu reduzieren, muss in vielen Bereichen der Umgang mit Kunststoffen anders gestaltet werden. Ein verbessertes Abfallmanagement bietet eine Option, ist allerdings eine sogenannte „end-of-pipe“-Lösung, also eine Lösung, die nur die Effekte aber nicht die Ursache bekämpft. Maßnahmen sollten ebenfalls bei der Ursache ansetzen und darauf abzielen, die Menge der Plastikverwendung und damit 
auch den Abfall zu reduzieren. Ein Feld der Intervention sind Verpackungen von Konsumgütern wie z. B. Lebensmitteln. Denn betrachtet man die Verwendung von Kunststoffen, machen Verpackungen mit fast $40 \%$ den größten Anteil der Kunststoffverwendung in Europa aus - ein Kunststoff mit einer nur sehr geringen Lebensdauer, der direkt nach seiner Verwendung entsorgt wird (PlasticsEurope 2016). Die Vorteile einer Kunststoffverpackung sind vielseitig: Das geringe Gewicht bedingt einen geringen Transportenergiebedarf, durch Verpackungen wird die Haltbarkeit von Lebensmittel erhöht, zudem sind Transparenz und vielfältige Formen der Verarbeitung möglich. Kein Wunder, dass sich die Kunststoffverpackung auf einem Siegeszug befindet: In den letzten 20 Jahren hat sich in Deutschland der private Verbrauch von Kunststoffverpackungen pro Kopf mehr als verdoppelt, von $12 \mathrm{~kg}$ pro Kopf im Jahr 1991 auf $24 \mathrm{~kg}$ pro Kopf im Jahr 2011 (Schüler 2015). Gründe für die Zunahme sind der steigende Verbrauch von Kunststoffflaschen und Kleinverpackungen, der Trend zu aufwändigeren Kunststoffverschlüssen und vorverpackter Thekenware wie Wurst und Käse in Dickfolien anstatt von Bedienungsware in Dünnfolie (Schüler 2015). Auch weisen inzwischen viele Supermärkte Regale mit gekühlten, verpackten Convenienceprodukten auf, wie geschnittenes Obst, Salate, Sandwiche oder Sushi. Ein weiterer Trend ist der Außer-Haus-Verzehr bedingt durch ein Angebot an Food-to-go-Produkten und ganze Supermärkte, die ihr Konzept danach ausrichten. Gleichzeitig verstärken aktuelle gesellschaftliche Entwicklungen den Trend zur Kunststoffverpackung (BVE 2016; Monkhouse et al. 2004). So führen der soziodemografische Wandel und die steigende Anzahl von Single- oder Zweipersonenhaushalten in der Tendenz zu kleineren Verpackungsgrößen und damit höherem Verpackungsverbrauch.

Eine Verbraucherstudie von PricewaterhouseCoopers zu Verpackungen legt jedoch auch ein wachsendes Umweltbewusstsein bei den Verbraucher*innen bezüglich Verpackungen nahe: $82 \%$ der Befragten, so die Studie, würden verpackungsfrei einkaufen, wenn es möglich wäre (pwc 2015). Bei der Frage, welche Steuerungsinstrumente eingesetzt werden können, um nachhaltige Konsum- und Produktionsmuster zu befördern, werden „harte“ Instrumente wie ökonomische und rechtliche gegenüber „,weichen“ wie Informations- und Kommunikationsstrategien als wirksamer angesehen (Weller 2013). Dies spiegelt auch die Einsicht wider, dass beim Thema Vermeidung von Plastikverpackungen die Verantwortung für ökologisch-nachhaltiges Handeln nicht allein auf die Verbraucher*innen abgewälzt werden darf, sondern dass bereits beim Hersteller und Handel angesetzt und hier nachhaltige Marktangebote geschaffen werden sollten (Ahaus et al. 2011). In diesem Innovationsbereich können Vermeidungsstrategien ansetzen, wobei, um 
das Problem möglichst effizient zu bearbeiten, die Bereiche Produktion und Konsum nicht getrennt voneinander betrachtet werden sollten (Marvin und Medd 2004).

Wir untersuchen daher genau diese Schnittstelle von Produktion und Konsum. Die Ausgangsthese hinter der Untersuchung ist, dass sich Produktion und Konsum von Lebensmitteln zunehmend trennen und ausdifferenzieren, wodurch ein höherer Bedarf an Verpackungen entsteht. Das bedeutet, dass zum einen die Produktion der Lebensmittel zunehmend aufwendiger wird und immer mehr Arbeitsschritte erfordert und zum anderen immer weitere Wege vom Produzenten bis zum Konsumenten zurückgelegt werden. Plastikverpackungen sind ein wichtiger Bestandteil vieler dieser räumlichen und zeitlichen Bearbeitungsschritte, erleichtern sie beispielsweise den Transport oder verbessern die Haltbarkeit der Lebensmittel (Sattlegger et al. 2020a). Um den Verbrauch von Verpackungen in diesen Bereichen genauer zu untersuchen, betrachten wir die Lebensmittelversorgungskette von den Produzenten bis zum Einzelhandel (Sattlegger 2020). Durch teilnehmende Beobachtung in der Lebensmittelproduktion, Großhandel und Einzelhandel wollen wir folgende Fragen beantworten: Welche Funktionen erfüllen Verpackungen im jeweiligen Unternehmen? Welche zentralen Handlungen innerhalb des Unternehmens sind an Verpackungen geknüpft? Wie verknüpfen Verpackungen die unterschiedlichen Unternehmen (Produktion bis Einzelhandel) entlang der Wertschöpfungskette miteinander? Welche verpackungsabhängigen Handlungsweisen vermitteln zwischen den Unternehmen - z. B. Transport oder Kommunikation? Damit möchten wir herausfinden, wie Plastikverpackungen verschiedene Aktivitäten und Praktiken in einer zunehmend globalisierten und (räumlich, zeitlich und sozial) ausdifferenzierten Lebensmittelversorgung verknüpfen. Letztendlich sollen Strategien erarbeitet werden, wie eine nachhaltigere und weniger materialintensive Reorganisation der Aktivitäten in der Lebensmittelversorgungskette aussehen kann, um den Plastikverpackungsmüll zu reduzieren.

\subsection{Biokunststoffe als Alternative:}

Kann man herkömmliche Kunststoffsorten ersetzen?

Der Verbrauch von Kunststoffverpackungen kann in einigen Bereichen zwar reduziert werden, doch werden Verpackungen auch in Zukunft eine wichtige Rolle spielen. Daher wird intensiv über nachhaltigere und ökologischere Alternativen nachgedacht. Dabei werden immer wieder sogenannte Biokunststoffe als möglicher Ersatz für konventionelle Kunststoffarten diskutiert. Der Begriff „Biokunststoff“" wird allerdings nicht einheitlich verwendet, sondern beschreibt eine 
recht große Gruppe von Kunststoffarten mit verschiedenen Eigenschaften: Entweder basieren diese auf nachwachsenden Rohstoffen und nicht auf Erdöl, sind dabei aber genauso schwer abbaubar wie herkömmliche Kunststoffe oder sie sind biologisch abbaubar und können dabei sowohl auf fossilen als auch auf regenerativen Rohstoffen basieren (Beier 2009). Ein Polymer ist laut Definition bioabbaubar, wenn mindestens ein Schritt während des Abbauprozesses von natürlich vorkommenden Organismen vollzogen wird. Der Abbau erfolgt in Gegenwart von Sauerstoff und Feuchtigkeit bei Umgebungstemperatur.

Der Großteil der verwendeten Verpackungen wird derzeit aus den konventionellen Polymeren Polyethylen (PE) und Polypropylen (PP) (zusammen ca. $85 \%$ ) hergestellt (Detzel et al. 2012). Will man die konventionell verwendeten Polymere durch abbaubare Biopolymere ersetzen, müssen die möglichen Alternativen dieselben Anforderungen wie das ursprünglich verwendete Material erfüllen, z. B. bestimmte Barriere-Eigenschaften. Auch muss sichergestellt sein, dass die Materialien nicht schon beginnen abzubauen, wenn sie noch im Einsatz, d. h. im Kontakt mit Lebensmitteln sind. Gängige bioabbaubare Kunststoffe auf dem Markt sind Polymilchsäure (PLA), Polycaprolacton (PCL) oder Polyhydroxybuttersäure (PHA).

Die Abbaubarkeit dieser Polymere ist allerdings umstritten bzw. es handelt sich bei den gängigen auf dem Markt erhältlichen Kunststoffarten zumeist um sogenannte „kompostierbare“ Materialien, was nicht mit der Definition für „bioabbaubar" gleichzusetzen ist. Denn diese Polymere können nur unter bestimmten Umweltbedingungen biologisch abgebaut werden, die im Grunde nur in industriellen Kompostieranlagen erreicht werden (Detzel et al. 2012). Somit ist ein Abbau weder auf heimischen Komposthaufen noch in der Umwelt zu erwarten, sondern es ist mit einer deutlich längeren Zerfallszeit zu rechnen (Haider et al. 2019a). Die Entsorgung solcher Verpackungen ist daher problematisch - diese gehören nicht in die Biotonne, können aber auch nicht gemeinsam mit konventionellen Kunststoffverpackungen entsorgt werden, da sie den Recyclingprozess der konventionellen Kunststoffarten stören. Diese Biokunststoffe müssen bisher über den Restmüll entsorgt werden und werden daher schlussendlich verbrannt (Völker und Kramm 2021).

Insgesamt ist im Verpackungsbereich eher ein Trend zu biobasierten, also auf erneuerbaren Ressourcen basierenden Kunststoffen und weniger zu bioabbaubaren Alternativen zu verzeichnen (Detzel et al. 2012). Ein Beispiel für solch einen biobasierten Kunststoff ist Bio-PE, welches z. B. aus Zuckerrohr gewonnen wird und dabei exakt dieselbe Molekülstruktur wie aus Erdöl hergestelltes PE besitzt und somit auch die gleiche Beständigkeit in der Umwelt aufweist. 
Die meisten aktuell produzierten Biokunststoffe schneiden in der Gesamtökobilanz nicht besser als herkömmliche Kunststoffe ab (Detzel et al. 2012) und können daher nicht generell als Lösungsstrategie verstanden werden. Es wird zudem die Sorge geäußert, dass Biokunststoffe beim Verbraucher ein falsches Entsorgungsverhalten befördern, da die Deklaration als abbaubare Kunststoffe implizieren kann, dass diese in die Umwelt entsorgt werden können. Aufgrund ihres derzeit noch geringen Marktanteils - weniger als 0,5 \% im Jahr 2009 (Detzel et al. 2012) - eignen sie sich zudem (noch) nicht für das Recyclingsystem. Dennoch wird Biokunststoffen in Zukunft ein großes Potenzial prognostiziert (European Bioplastics 2015) und sie können als Teillösung für bestimmte Bereiche mitgedacht werden. So könnten sie durchaus als Ersatzstoff in einigen Produkten dienen, die besonders leicht in die Umwelt gelangen. Hierfür müssen die Kunststoffe vor allem hinsichtlich ihrer Abbaubarkeit in der Umwelt weiter optimiert werden. Zudem erfordert die Verknappung fossiler Rohstoffe konventionelle Materialien aus nachwachsenden Rohstoffen herzustellen, weshalb biobasierte Kunststoffe langfristig ohnehin die konventionellen Kunststoffarten ersetzen müssen.

Wir untersuchen Biokunststoffe deshalb aus beiden Richtungen: Zum einen gehen wir der Frage nach, wie man die Abbaubarkeit von Kunststoffen weiter verbessern kann. Hierfür versuchen wir ein Polymer zu synthetisieren, dass die Eigenschaften von PE imitiert, also sich prinzipiell für die Verwendung als Verpackungsmaterial eignen würde, und gleichzeitig bioabbaubar ist (Haider et al. 2019b). Zum anderen wird untersucht, wie PE basierend aus dem nachwachsenden Rohstoff Lignin hergestellt werden kann, welches als Abfallprodukt der Papier- und Holzindustrie erhältlich ist und damit nicht in Konkurrenz zur Nahrungsmittelproduktion steht.

\section{Zusammenarbeit mit Partnern aus der Praxis}

Im Forschungsprozess arbeiten wir mit sehr unterschiedlichen Partnern aus der gesellschaftlichen Praxis zusammen, die, wie im Forschungsdesign angelegt, eine möglichst breite Sicht auf das Problemfeld abdecken. In unseren Arbeiten unterstützen uns Vertretern aus der Plastikindustrie, dem Einzelhandel, dem Umweltschutz, der Wasser- und Abfallwirtschaft, der Entwicklungszusammenarbeit und aus Umweltbehörden. Gemeinsam diskutieren wir mit den Praxispartnern die unterschiedlichen Lösungsstrategien und erörtern die jeweiligen Perspektiven und Problemwahrnehmungen. In einer ersten Diskussionsrunde zeigte sich, dass alle Partner, ob aus Industrie oder Umweltschutz, den Eintrag von Plastikmüll 
in die Umwelt als problematisch ansehen und ein Entgegensteuern befürworten. Deutliche Diskrepanzen ergaben sich allerdings bei der Frage, wie mit dem Material Plastik in Zukunft umzugehen ist. Von der einen Seite wurde die Persistenz des Materials als problematisch hervorgehoben und über eine Vermeidung bis hin zum Verbot diskutiert. Auf Handels- und Industrieseite standen die Vorteile des Materials bzw. seine Unersetzbarkeit in vielen Bereichen im Vordergrund und mögliche Lösungen zielten auf verbessertes Abfallmanagement und Recycling. Im nächsten Schritt sollen mit den Partnern unterschiedliche Lösungsstrategien, die an verschiedenen Stellen im Produktzyklus ansetzen, hinsichtlich ihrer Umsetzbarkeit und möglicher Nebenfolgen abgewogen werden.

Neben den breiteren Diskussionsrunden wird in den verschiedenen Forschungsfeldern enger mit unterschiedlichen Praxispartnern kooperiert, um das Wissen aus der gesellschaftlichen Praxis in die Forschung einzubinden und die Ergebnisse für die Praxis nutzbar zu machen (siehe z. B. Sattlegger et al. 2020b). So arbeiten wir bei der Risikobewertung von Mikroplastik mit Experten aus Umwelt- und Wasserbehörden zusammen. Die Untersuchung der MeeresmüllProblematik anhand von Fallstudien in Asien erfolgt gemeinsam mit zwei Organisationen der internationalen Zusammenarbeit aus Umwelt und Entwicklung und Ergebnisse zur Vermeidung und Substitution herkömmlicher Kunststoffverpackungen werden mit Partnern aus Handel und Industrie diskutiert.

\section{$4 \quad$ Integrative Betrachtung der unterschiedlichen Forschungsfelder}

Aus der systemischen Perspektive werden die Ergebnisse der Gruppenmitglieder diskutiert und aufeinander bezogen, wodurch die Zusammenhänge zwischen den einzelnen Bereichen deutlich werden. Hieraus ergeben sich Problematiken und neue Fragestellungen, die durch eine rein disziplinäre Bearbeitung der Thematik nicht betrachtet werden. Am Beispiel der Mikroplastik-Thematik zeigt sich, dass in Medien und Politik zumeist primäres Mikroplastik adressiert und die Kosmetikindustrie als Hauptverursacher ausgemacht wird (Kramm und Völker 2018). Doch ist inzwischen bekannt, dass es sich bei dem Großteil des Plastiks in den Weltmeeren um sekundäres Mikroplastik handelt, also Fragmente, die durch den Zerfall von größerem Plastikabfall entstanden sind. Das bedeutet, dass primäres Mikroplastik aus Kosmetik nur einen verschwindend geringen Teil der Gesamtmenge ausmacht. Diese Tatsache spricht zwar nicht gegen ein Verbot von Mikroplastik in Kosmetik, da es sich um einen leicht zu vermeidenden Eintrag handelt, denn Mikroplastik kann einfach durch natürliche, weniger umstrittene 
Materialien ersetzt werden. Es zeigt aber, dass die Mikroplastik-Debatte nicht von der Debatte um größeren Plastikmüll in den Meeren entkoppelt werden darf und weitere Verursacher und Lösungsansätze parallel betrachtet werden müssen. Auch bei den Ansätzen zur Vermeidung weiterer Einträge von Plastikmüll in die Meere darf nicht allein Asien als Hauptverursacher in die Verantwortung genommen werden (Kramm und Völker 2017). Am Beispiel Tourismus wird deutlich, dass auch die Bevölkerung, die aus einer Region mit gutem Abfallmanagement kommt, als Verursacher in die Problematik eingebunden ist. Daher sind die Forschungsergebnisse auch für die deutsche Öffentlichkeit relevant. Zudem stammt ein Großteil der weltweit vertriebenen Plastikprodukte und damit auch der Abfälle in der Umwelt aus westlichen Konzernen. Hier stellt sich die Frage, wie viel Verantwortung die Hersteller eines Produktes letztendlich auch für seine korrekte Entsorgung übernehmen müssen.

Wenn es um alternative Materialien wie Bioplastik geht, dürfen sich die ökologischen Vorteile nicht auf eine bessere Abbaubarkeit in der Umwelt beschränken, sondern müssen andere Bereiche, wie z. B. den Energiebedarf bei der Produktion, einschließen. Hier bleibt fraglich, ob es generell der richtige Weg ist, Materialien herzustellen, die nur eine kurze Lebensdauer besitzen - egal ob diese bioabbaubar sind oder nicht. Demgegenüber stehen die deutlich verlängerte Haltbarkeit von verpackten Lebensmitteln und damit eine Vermeidung von Lebensmittelabfällen. Doch auch hier stellt sich die Frage, ob die derzeitige Art der Lebensmittelversorgung, die zum Teil von Überproduktion und langen Transportwegen gekennzeichnet ist, nicht nachhaltiger zu gestalten ist und damit einerseits Verpackungen überflüssig werden und andererseits auch weitere positive Aspekte für die Umwelt erreicht werden können.

Das Plastikbeispiel zeigt, dass es in vielen Bereichen um die generelle Frage geht, wie sich die Gesellschaft in Zukunft nachhaltig entwickeln kann. Auf einer übergeordneten Ebene stellen wir uns daher die Fragen, wie von Gesellschaften produzierte, systemische Risiken adäquat eingeschätzt und bewertet und trotz bestehender Unsicherheiten Maßnahmen beschlossen und umgesetzt werden können. Damit möchten wir Impulse für eine integrative sozial-ökologische Risikoforschung setzen (Völker et al. 2017).

Danksagung Unser Dank gilt den Doktorand*innen der Nachwuchsgruppe PlastX Tobias Haider, Heide Kerber, Lukas Sattlegger und Lisa Zimmermann für die erfolgreiche Zusammenarbeit. Weiterhin möchten wir dem BMBF für die Finanzierung unserer Forschung danken. 


\section{Literatur}

Ahaus, B., L. Heidbrink, und I. Schmidt. 2011. Der verantwortliche Konsument: Wie Verbraucher mehr Verantwortung für ihren Alltagskonsum übernehmen können. Working papers des CRR 10. Essen: Kulturwissenschaftliches Institut.

Andrady, A. L. 2011. Microplastics in the marine environment. Marine Pollution Bulletin 62 (8):1596-1605.

Andrady, A. L., und M. A. Neal. 2009. Applications and societal benefits of plastics. Philosophical Transactions of the Royal Society B: Biological Sciences 364 (1526):1977-1984.

Andrady, A. L., M. Bomgardner, D. Southerton, C. Fossi, und A. Holmström. 2015. Plastics in a sustainable society. Background paper. The swedish foundation for strategic environmental research. Stockholm: MISTRA.

Ballance, A., P. G. Ryan, und J. K. Turpie. 2000. How much is a clean beach worth? The impact of litter on beach users in the Cape Peninsula, South Africa. South African Journal of Science 96 (5):210-230.

Bechmann, G., F. Keil, K. Kümmerer, und E. Schramm. 2007. Systemische Risiken aus sozial-ökologischer Perspektive. Start-Impulspapier 05:1-7.

Becker, E., und T. Jahn. 2006. Soziale Ökologie - Grundzüge einer Wissenschaft von den gesellschaftlichen Naturverhältnissen. Frankfurt a. M.: Campus.

Beier, W. 2009. Biologisch abbaubare Kunststoffe. UBA Hintergrundpapier. Dessau: Umweltbundesamt.

Besseling, E., B. Wang, M. Lürling, und A. A. Koelmans. 2014. Nanoplastic affects growth of $S$. obliquus and reproduction of D. Magna. Environmental Science and Technology 48 (20):12336-12343.

Browne, M. A., P. Crump, J. S. Niven, E. Teuten, A. Tonkin, T. Galloway, und R. Thompson. 2011. Accumulation of microplastic on shorelines worldwide: Sources and sinks. Environmental Science \& Technology 45 (21):9175-9179.

Bundesinstitut für Risikobewertung. 2016. BfR-Verbrauchermonitor 0212016. Berlin: BfR.

Bundesvereinigung der Deutschen Ernährungsindustrie (BVE), Hrsg. 2016. FAKT: ist. Teil 3 Lebensmittelverpackung - Von der Entsorgung zum Recycling. Berlin: BVE.

Der Spiegel. 2013. Plastikteilchen verunreinigen Lebensmittel: Unterschätzte Gefahr. https:// www.spiegel.de/wissenschaft/technik/winzige-plastikteile-verunreinigen-lebensmittel-a934057.html. Zugegriffen: 15. Juni 2020.

Derraik, J. G. B. 2002. The pollution of the marine environment by plastic debris: A review. Marine Pollution Bulletin 44 (9):842-852.

Detzel, A., B. Kauertz, C. Derreza-Greeven, und A. Kirsch. 2012. Untersuchung der Umweltwirkungen von Verpackungen aus biologisch abbaubaren Kunststoffen. Dessau-Roßlau: Umweltbundesamt.

Eerkes-Medrano, D., R. C. Thompson, und D. C. Aldridge. 2015. Microplastics in freshwater systems: A review of the emerging threats, identification of knowledge gaps and prioritisation of research needs. Water Research 75:63-82.

Europäische Kommission. 2013. Grünbuch zu einer europäischen Strategie für Kunststoffabfälle in der Umwelt. Brüssel: Europäische Kommission.

European Bioplastics. 2015.Was sind Biokunststoffe? Begriffe, Werkstofftypen und Technologien - eine Einführung. https://www.petroplast.ch/fileadmin/pdf/HOI_Biokunststoffe_ 120911.pdf. Zugegriffen: 15. Juni 2020. 
European Chemicals Bureau. 2003. Technical guidance document on risk assessment. Part II. Luxemburg: Office for Official Publications of the European Communities.

Gregory, M. R. 1996. Plastic 'scrubbers' in hand cleansers: A further (and minor) source for marine pollution identified. Marine Pollution Bulletin 32 (12):867-871.

Gregory, M. R. 2009. Environmental implications of plastic debris in marine settings —entanglement, ingestion, smothering, hangers-on, hitch-hiking and alien invasions. Philosophical Transactions of the Royal Society B: Biological Sciences 364 (1526):2013-2025.

Haider, T., C. Völker, J. Kramm, K. Landfester, und F. R. Wurm. 2019a. Plastics of the future? The impact of biodegradable polymers on the environment and on society. Angewandte Chemie International Edition 58 (1):50-62.

Haider, T., O. Shyshov, O. Suraeva, I. Lieberwirth, M. von Delius, und F. R. Wurm. 2019b. Long-Chain polyorthoesters as degradable polyethylene mimics. Macromolecules 52 (6):2411-20. 2411-2420.

Jaeger-Erben, M., J. Kramm, M. Sonnberger, C. Völker, C. Albert, A. Graf, K. Hermans, S. Lange, T. Santarius, B. Schröter, S. Sievers-Glotzbach, und J. Winzer. 2018. Building capacities for transdisciplinary research. Challenges and recommendations for early-career researchers. GAIA - Ecological Perspectives for Science and Society 27 (4):379-386.

Jambeck, J. R., R. Geyer, C. Wilcox, T. R. Siegler, M. Perryman, A. Andrady, R. Narayan, und K. L. Law. 2015. Plastic waste inputs from land into the ocean. Science 347 (6223):768771.

Jang, Y. C., S. Hong, J. Lee, M. J. Lee, und W. J. Shim. 2014. Estimation of lost tourism revenue in Geoje Island from the 2011 marine debris pollution event in South Korea. Marine Pollution Bulletin 81 (1):49-54.

Kerber, H., und J. Kramm. 2020. Der Müll in unseren Meeren: Ursachen, Folgen, Lösungen. Geographische Rundschau 7 (8):16-20.

Kerber, H., und J. Kramm. 2021. On- and offstage: Encountering entangled waste-tourism relations on the vietnamese Island of Phu Quoc. The Geographical Journal. https://doi. org/10.1111/geoj.12376.

Koelmans, A. A., E. Besseling, E. Foekema, M. Kooi, S. Mintenig, B. C. Ossendorp, P. E. Redondo-Hasselerharm, A. Verschoor, A. P. van Wezel, und M. Scheffer. 2017. Risks of plastic debris: Unravelling fact, opinion, perception, and belief. Environmental Science \& Technology 51 (20):11513-19.

Kramm, J., und C. Völker. 2017. Plastikmüll im Meer: Zur Entdeckung eines Umweltproblems. APuZ - Aus Politik und Zeitgeschichte 67 (51-52):17-22.

Kramm, J., und C. Völker. 2018. Understanding the risks of microplastics: A social-ecological risk perspective. In Freshwater microplastics. Emerging environmental contaminants, Hrsg. M. Wagner und S. Lambert. The handbook of environmental chemistry 58:223237. Cham: Springer International Publishing.

Kramm, J., C. Völker, und M. Wagner. 2018. Superficial or substantial: Why care about microplastics in the anthropocene? Environmental Science \& Technology 52 (6):33363337.

Lu, Y., Y. Zhang, Y. Deng, W. Jiang, Y. Zhao, J. Geng, L. Ding, und H. Ren. 2016. Uptake and accumulation of polystyrene microplastics in zebrafish (danio rerio) and toxic effects in liver. Environmental Science \& Technology 50 (7):4054-4060.

Macarthur, D. E., D. Waughray, und R. Stutchtey. 2016. The new plastics economy: Rethinking the future of plastics. Cology: World Economic Forum. 
Marvin, S., und W. Medd. 2004. Sustainable infrastructures by proxy? Intermediation beyond the production-consumption nexus. In Sustainable consumption: The implications of changing infrastructures of provision, Hrsg. D. Southerton, H. Chappells, und B. Van Vliet, 81-96. Cheltenham: Elgar.

Monkhouse, C., C. Bowyer, und A. Farmer. 2004. Packaging for sustainability. Packaging in the context of the product, supply chain and consumer needs. IEEP Report for INCPEN. Institute for European Environmental Policy.

Nash, A. D. 1992. Impacts of marine debris on subsistence fishermen an exploratory study. Marine Pollution Bulletin 24 (3):150-156.

NDR. 2014. Mikroplastik in Mineralwasser und Bier. https://www.presseportal.de/pm/6561/ 2750798. Zugegriffen: 19. Juni 2020

OECD. 2003. Emerging risks in the 21 st century: an agenda for action. Paris: OECD.

Oliveira, M., A. Ribeiro, K. Hylland, und L. Guilhermino. 2013. Single and combined effects of microplastics and pyrene on juveniles ( $0+$ Group) of the common goby pomatoschistus microps (Teleostei, Gobiidae). Ecological Indicators 34:641-647.

PlasticsEurope. 2016. Plastics - the facts 2016. An analysis of European plastics production, demand and waste data. https://www.plasticseurope.org/application/files/4315/1310/ 4805/plastic-the-fact-2016.pdf. Zugegriffen: 19. Juni 2020.

pwc. 2015. Verpackungsfreie Lebensmittel - Nische oder Trend? Verbraucherbefragung. https://www.pwc.de/de/handel-und-konsumguter/assets/pwc-verpackungsfreie-leb ensmittel.pdf. Zugegriffen: 15. Juni 2020.

Rehse, S., W. Kloas, und C. Zarfl. 2016. Short-term exposure with high concentrations of pristine microplastic particles leads to immobilisation of daphnia magna. Chemosphere 153:91-99.

Sattlegger, L., I. Stieß, L. Raschewski, und K. Reindl. 2020a. Plastic packaging, food supply and everyday life: Adopting a social practice perspective in social-ecological research. Nature and Culture. 15 (2):146-172.

Sattlegger, L., L. Zimmermann, und M. Birnbach. 2020b. Von der unsichtbaren zur durchschaubaren Verpackung Prinzipien nachhaltiger Verpackungsgestaltung. Ökologisches Wirtschaften 35 (1):38-42.

Sattlegger, L. 2020. Making food manageable - Packaging as a code of practice for work practices at the supermarket. Journal of Contemporary Ethnography. 50 (3): 341-367.

Schüler, K. 2015. Aufkommen und Verwertung von Verpackungsabfällen in Deutschland im Jahr 2013. Dessau-Roßlau: Umweltbundesamt.

Stöfen-O'Brien, A. 2015. The international and european legal regime regulating marine litter in the EU. Baden-Baden: Nomos.

Teuten, E. L., S. J. Rowland, T. S. Galloway, und R. C. Thompson. 2007. Potential for plastics to transport hydrophobic contaminants. Environmental Science \& Technology 41 (22):7759-7764.

UNEP. 2016. Marine plastic debris and microplastics -Global lessons and research to inspire action and guide policy change. Nairobi: United Nations Environment Programme.

Velis, C. A. 2014. Global recycling markets: Plastic waste. A story for one player - China. Report prepared by FUELogy and formatted by D-waste on behalf of international solid waste association - Globalisation and waste management task force. Vienna: ISWA. 
Völker, C., J. Kramm, H. Kerber, E. Schramm, M. Winker, und M. Zimmermann. 2017. More than a potential hazard-Approaching risks from a social-ecological perspective. Sustainability 9 (7):1039.

Völker, C., und J. Kramm. 2020. Bioplastik - Kunststoffe der Zukunft? In Einfach weglassen? Ein wissenschaftliches Lesebuch zur Reduktion von Plastikverpackungen im Lebensmittelhandel, Hrsg. M. Kröger, J. Pape, und A. Wittwer, 393-407. München: oekom verlag.

Völker, C., J. Kramm, und M. Wagner. 2019. On the creation of risk: Framing of microplastics risks in science and media. Global Challenges. https://doi.org/10.1002/gch2.201900010.

Wagner, M., C. Scherer, D. Alvarez-Muñoz, N. Brennholt, X. Bourrain, S. Buchinger, E. Fries, u. a. 2014. Microplastics in freshwater ecosystems: What we know and what we need to know. Environmental Sciences Europe 26 (1):12.

Wissenschaftlicher Beirat der Bundesregierung Globale Umweltveränderung (WBGU). 2013. Welt im Wandel: Menschheitserbe Meer. Hauptgutachten. Berlin: WBGU.

Weller, I. 2013. Nachhaltiger Konsum, Lebensstile und Geschlechterverhältnisse. In Geschlechterverhältnisse und Nachhaltigkeit, Hrsg. S. Hofmeister, C. Katz, und T. Mölders, 286-295. Opladen: Budrich.

Wright, S. L., R. C. Thompson, und T. S. Galloway. 2013. The physical impacts of microplastics on marine organisms: A review. Environmental Pollution 178:483-492.

Zimmermann, L., S. Göttlich, J. Oehlmann, M. Wagner, und C. Völker. 2020. What are the drivers of microplastic toxicity? Comparing the toxicity of plastic chemicals and particles to Daphnia magna. Environmental Pollution 267 (115392).

Zitko, V., und M. Hanlon. 1991. Another source of pollution by plastics: Skin cleaners with plastic scrubbers. Marine Pollution Bulletin 22 (1):41-42.

Johanna Kramm, Dr., Leiterin der Nachwuchsgruppe PlastX, ISOE - Institut für sozialökologische Forschung, Frankfurt am Main.

https://www.isoe.de/forschung/nachwuchsgruppe/

Carolin Völker, Dr., Leiterin der Nachwuchsgruppe PlastX, ISOE - Institut für sozialökologische Forschung, Frankfurt am Main.

https://www.isoe.de/forschung/nachwuchsgruppe/ 
Open Access Dieses Kapitel wird unter der Creative Commons Namensnennung 4.0 International Lizenz (http://creativecommons.org/licenses/by/4.0/deed.de) veröffentlicht, welche die Nutzung, Vervielfältigung, Bearbeitung, Verbreitung und Wiedergabe in jeglichem Medium und Format erlaubt, sofern Sie den/die ursprünglichen Autor(en) und die Quelle ordnungsgemäß nennen, einen Link zur Creative Commons Lizenz beifügen und angeben, ob Änderungen vorgenommen wurden.

Die in diesem Kapitel enthaltenen Bilder und sonstiges Drittmaterial unterliegen ebenfalls der genannten Creative Commons Lizenz, sofern sich aus der Abbildungslegende nichts anderes ergibt. Sofern das betreffende Material nicht unter der genannten Creative Commons Lizenz steht und die betreffende Handlung nicht nach gesetzlichen Vorschriften erlaubt ist, ist für die oben aufgeführten Weiterverwendungen des Materials die Einwilligung des jeweiligen Rechteinhabers einzuholen. 